\title{
La calidad del control prenatal como garantía de salud de madres y recién nacidos
}

\author{
Sonia Pazmiño de Osorio*; Farid Amashta; Carlos Perdomo; Luis E. Torres; José Rojas; demás colaboradores**; María \\ Cecilia Arturo Rojas***; Luis Zambrano; Karl Narjer; Vlasco Juvinao; Carlos Agudelo****
}

\section{RESUMEN}

Con el objetivo de validar los buenos resultados en la salud materna y del recién nacido obtenidos con un programa de Mejoramiento Continuo de la Atención Prenatal realizado en instituciones de salud de la ciudad de Cali en 1994 - 1995 se vincularon 15 instituciones de las comunas 6,7 y 14 de la ciudad (Agosto / 96 - Octubre /97) y siete (7) hospitales de las ciudades de Cartago, Zarzal, Tuluá, Guacarí, Palmira, Yumbo y Jamundí del Departamento del Valle (Setiembre /96 - Noviembre /97). La metodología del programa fue similar.

El modelo de control de calidad se basó en la vigilancia del cumplimiento en las normas institucionales de atención a las gestantes, usando como instrumento 102 variables de la historia clínica. Un gineco-obstetra lo aplicó semanalmente en una muestra del 7.2 de las gestantes. Esto permitió evaluar el desempeño de médicos y auxiliares, y con base a los defectos encontrados darles capaciéación.

Al aplicar una metodología de investigación, acción, participación con los directivos y el personal operativo, se tomaron los correctivos en forma oportuna.

Se demuestra objetivamente la mejoría de la calidad de atención.

Hay una disminución progresiva de los defectos mes a mes hasta llegar al $100 \%$ de cero defectos, que se mantuvo durante el año del estudio.

Los resultados en la salud de madres y recién nacidos confirman los hallazgos del primer estudio.

Hubo un mayor diagnóstico y manejo de la patología materna, la ruptura prematura de membranas y la amenaza de parto prematuro disminuyeron a la mitad, no hubo muertes maternas atribuibles a la atención prenatal; disminuyó la patología del recién nacido como prematurez $41.6 \%$ y la mortalidad fetal $95 \%$.

Se concluye que el desarrollo de modelos de control de calidad similares, son una estrategia para disminuir la morbi-mortalidad materna y perinatal.

PALABRAS CLAVES: Control prenatal, control de calidad, mortalidad materna, mortalidad prenatal.

\section{SUMMARY}

Fifteen Primary Health Centres, in Cali (Colombia) belong to the lower socioeconomic strata, and seven Hospitals of the Valle del Cauca state, were enrolle in an extensive continuous quality improvement programme aiming to reach effectiveness of child and maternal health. The methodology in order to improve ploformance was similar for all institutions and it has been published elsewhere.

Quality and approaches to its assessment were based on precriptive and explicit criteria of prenatal cara programme using 102 items of the clinical chart. A trainet Obstetrician / Gynecologist applied this instrument, weekly, on a sample size of $7.2 \%$ of the whole pregnant women attending to this public health network.

The monitoring of the physicians and auxiliary nurses work permitted to evaluate their appropiate perfomance and providing them with the skills to implement their quality evaluation cycle.

During the intervention process a significantly reduction of the number of "chart defects" were observed becoming of cero defects three months later.

After one year period the findings confirmed the previous outcomes: an increase of the percentage of maternal pathologies diagnosed; premature rupture of the membranes and threatened premature delivery felt down one half those rates, previously reported.

Newborn pathology in special preterm birth decreased to $41.6 \%$, and fetal mortality $95 \%$. No maternal deaths were observed during this period of time.

Definitely the quality assessment and assurance of the prenatal control constitute one of the best strategy to reduce maternal and child mortality and morbility.

KEY WORDS: Quality assessment, continuous quality improvement, assurance, prenatal mortality, maternal deaths.

* Gineco-Obstetra y Salubrista. Directora del Programa.

** Sociedad Vallecaucana de Obstetricia y Ginecología (SOVOGIN)
Presidente Sociedad Vallecaucana de Obstetricia y Ginecología (SOVOGIN).

Gineco-Obstetras de SOVOGIN 
Desde hace varias décadas en Colombia se han realizado diferentes actividades para intentar bajar la mortalidad materna siendo una de las más importantes el incremento de cobertura en la atención prenatal y en la atención del parto institucional que ha variado del 30 $40 \%$ en la década del 70 , al 70-80\% respectivamente en la década del 90; en algunas ciudades como Cali se acercó al $90 \%$.

El modelo de atención se sustentó en personal auxiliar de enfermería y en el médico general; los resultados no han sido satisfactorios, pues de acuerdo con la OMS las cifras permanecen estables en $140 \times 100.000 \mathrm{nv}$ desde hace 12 años (1).

Salazar Ana encontró que el $48 \%$ de las muertes maternas en Cali tenían control prenatal (2) y La Sociedad Vallecaucana de Obstetricia y Ginecología decidió evaluar los resultados de una atención prenatal con calidad, de acuerdo con las normas, a través de un modelo de control, capacitación y vigilancia durante el proceso con la participación de gineco-obstetras en el nivel primario con funciones de asesoría, control y capacitación.

Los resultados de esta experiencia en 1995 en 4 instituciones demuestran una notoria disminución en la morbilidad y mortalidad materna y del recién nacido (3).

Con el propósito de validar estos resultados se amplió el programa a 15 instituciones de las comunas 6,7 y 14 de la ciudad de Cali en el período comprendido entre Agosto 196 a Octubre 197 y a 7 hospitales de las ciudades de Cartago, Zarzal, Tuluá, Guacarí, Palmira, Yumbo y Jamundí del departamento del Valle en el período de Septiembre /96 a Noviembre / 97. Duración total 15 meses.

\section{Metodología}

Durante un período inicial de tres meses se hizo observación crítica del desempeño y calidad del cuidado médico a través del análisis de la historia clínica, del modelo de atención prenatal, de las normas del programa y revisión del estado y funcionamiento de los instrumentos de medición, (metros, tensiómetros y pesas). También se evaluaron el recurso humano, el apoyo médico y la prevalencia de período de las patologías.

Se encontró que las condiciones de los consultorios prenatales no permitían el examen físico completo ni siquiera el examen ginecológico.

Se inició la adecuación de los consultorios, se hizo el mantenimiento preventivo y la calibración de los instrumentos de medición, y se capacitó al personal médico y de enfermería en las normas de atención, incluyendo la estandarización de las técnicas de medición.

Se aplicó el mismo instrumento de observación del cumplimiento de las normas durante el proceso de atención utilizado en la investigación de 1995, incrementando la observación a 102 variables de la historia perinatal del CLAP (Centro Latinoamericano de Perinatología) de manera sistematizada.

A partir del cuarto mes un gineco-obstetra visitó los centros de atención una vez por semana, días diferentes, alternando horas de la mañana o de la tarde, de acuerdo al horario de consulta. Seleccionó al azar 10 historias clíni- cas de las gestantes (muestra del 5\%) y las revisó registrando los defectos en el instrumento de monitoreo diseñado, asignando un puntaje a cada variable según el llenado de la casilla, la detección del riesgo y la conducta seguida.

En los casos del manejo inadecuado se le dio capacitación al médico tratante.

Los puntajes de los defectos ponderados y acumulativos de las historias revisadas fueron condensados mensualmente y en reuniones periódicas, con directivos y personal operativo de los Centros, se analizaron y se tomaron las medidas necesarias para corregir los defectos. El porcentaje de cero defectos corresponde al total de variables de las historias revisadas que tenían buen diligenciamiento, detección del riesgo y conducta adecuada. Ideal ciento por ciento.

Semanalmente o cuando fue necesario se calibraron los tensiómetros y balanzas.

En las clínicas u hospitales de remisión se estableció vigilancia para el llenado completo en la historia clínica de toda la información respecto al parto, haciendo énfasis en las complicaciones perinatales.

En esta oportunidad se introdujo un sub-programa de pre-eclampsia que comprendió la detección de gestantes con factores de riesgo para desarrollar la enfermedad, educación con talleres de autocuidado, vigilancia, seguimiento en casa, otros niveles de atención y control postparto entre 3 y 6 meses después.

Para el análisis de resultados en la Salud de la Madre y el recién nacido se determinaron, tanto para el Valle como para Cali, las incidencias de las patologías por trimestre, desde el trimestre previo a la instalación del programa TriP1 y durante los trimestres 2,3,4 y 5 (un año) de funcionamiento vigilado del programa (Tri1, Tri2, Tri3, Tri4 y Tri5).

Las pruebas de significancia (Chi cuadrado) miden la variación entre las incidencias de las patologías entre el trimestre previo (TriP1) contra (TriP5).

\section{Población de estudio}

Todas las gestantes que asistieron, durante el período de estudio, a control prenatal en los centros de salud de la Secretaría de Salud Pública, en las comunas 6,7 y 14 de Cali y 7 hospitales del Departamento del Valle, Cartago, Zarzal, Tuluá Guacarí, Yumbo, Palmira y Jamundí.

Las gestantes que asisten a estas instituciones pertenecen a un estrato socioeconómico bajo. El control prenatal fue realizado por médicos generales y auxiliares de enfermería.

\section{Resultados del monitoreo}

De 65.710 consultas de las gestantes que asistieron a control fueron monitoreadas 4.736 historias $(7.2 \%)$ : 2.715 en Cali y 2.021 en los hospitales del Valle.

Al comienzo del programa la calidad de la atención medida a través del monitoreo de historias era inapropiada $60 \%$ de cero defectos y menos; en los meses siguientes se observó un rápido incremento hasta superar el $90 \%$ que se mantuvo el resto del año. 
Tabla 1

CERO DEFECTOS EN EXAMEN CLINICO Y DIAGNOSTICO

\begin{tabular}{|lrcc|}
\hline MES & ZARZAL & JAMUNDI & GUACARI \\
\hline ENERO & & & \\
FEBRERO & 59.1 & 41.6 & 65.4 \\
MARZO & 74.8 & 67.1 & 94.0 \\
ABRIL & 98.2 & 76.2 & 72.6 \\
MAYO & 100.0 & 83.6 & 84.5 \\
JUNIO & 100.0 & 90.0 & 99.3 \\
JULIO & 100.0 & 93.6 & 94.6 \\
AGOSTO & 100.0 & 99.3 & 92.6 \\
SEPTIEMBRE & 100.0 & 100.0 & 98.2 \\
OCTUBRE & 100.0 & 100.0 & 94.2 \\
\hline
\end{tabular}

Uno de los defectos más protuberantes fue la falta de examen a las gestantes. Esta labor de exclusiva responsabilidad médica se dificultaba por la falta de equipos, materiales y suministros. Las medidas tomadas se ven reflejadas en mejoramiento sostenido hasta $94-100 \%$. Tabla 1.

Además se mejoró la detección del riesgo obstétrico y la calidad de conductas tomadas.

En conclusión el modelo de control de calidad diseñado permitió cuantificar el mejoramiento en la calidad de atención, hizo énfasis en la detección y tratamiento oportuno de la morbilidad materna y en la detección de las principales patologías causantes de mortalidad materna y fetal como toxemia y prematurez. Fue notable la influencia del gineco-obstetra en la asesoría y capacitación de los médicos generales y el personal de enfermería.

\section{Resultados sobre la madre y el recién nacido}

Terminaron su embarazo 5.471 gestantes, de las cuales $6.8 \%$ en Cali y $7.5 \%$ en los Hospitales del Valle eran de alto riesgo según la edad (menores de 15 o mayores de 35 años); analfabetas $3.5 \%$ en Cali y $3.1 \%$ en el Valle.

Con índices educativos similares, primaria $38.6 \%$ y $36 \%$ secundaria 54.6 y $56.6 \%$ en Cali y Valle respectivamente.

Solteras $28.6 \%$ en Cali, y $18 \%$ en el Valle.

Se incrementó en general el diagnóstico y tratamiento oportuno de diabetes, hipertensión y la infección urinaria. Esta última fue significante en el total de casos y en el Valle del Cauca. Tabla 2. Gráfica 2.

Se incrementó el diagnostico y tratamiento oportuno de la anemia en Cali 46.7\%. Tabla 3. Gráfica 3.

Tanto en los hospitales del Valle como en Cali se encontró una disminución significante estadísticamente en la Preeclampsia de $53.1 \%$ en los primeros y $60.2 \%$ en Cali con una disminución concomitante de los casos de Eclampsia. Tabla 4. Gráfica 4.

La ruptura prematura de membranas disminuyó en los hospitales del Valle 56.4\%. Tabla 5. Gráfica 5.

La amenaza de parto prematuro disminuyó en el Valle $51.7 \%$ y en Cali $46.7 \%$ Tabla 6. Gráfica 6.

El parto prematuro disminuyó en el Valle $41.6 \%$, en Cali $42.4 \%$ Tabla 7. Gráfica 7. Hallazgos similares reportan Gómez y colaboradores en España (4).
Tabla 2

CAMBIOS EN LA INCIDENCIA DE INFECCION URINARIA

\begin{tabular}{|c|c|c|c|c|c|c|c|c|c|}
\hline & \multicolumn{3}{|c|}{ VALLE } & \multicolumn{3}{|c|}{ CALI } & \multicolumn{3}{|c|}{ TOTAL } \\
\hline & $\mathrm{N}$ & $\mathrm{n}$ & $\%$ & $\mathrm{~N}$ & $\mathrm{n}$ & $\%$ & $\mathrm{~N}$ & $\mathrm{n}$ & $\%$ \\
\hline Tri P1 & 503 & 54 & 10.7 & 178 & 27 & 15.3 & 681 & 81 & 11.9 \\
\hline Tri 2 & 597 & 70 & 11.7 & 315 & 59 & 18.7 & 912 & 129 & 14.1 \\
\hline Tri 3 & 636 & 107 & 16.8 & 496 & 114 & 23 & 1.132 & 221 & 19.5 \\
\hline Tri 4 & 780 & 139 & 17.8 & 459 & 108 & 23.5 & 1.239 & 247 & 19.9 \\
\hline Tri 5 & 832 & 117 & 14.1 & 411 & 75 & 18.2 & 1.243 & 191 & 15.4 \\
\hline Total & 3.612 & 2 517 & 14.3 & 1.859 & 383 & 20.6 & 5.471 & 900 & 16.5 \\
\hline \multicolumn{3}{|c|}{ Diferencia } & 24.1 & & & & & & 22.7 \\
\hline
\end{tabular}

Tabla 3

CAMBIOS EN LA INCIDENCIA DE LA ANEMIA

\begin{tabular}{|c|c|c|c|c|c|c|c|c|c|}
\hline & \multicolumn{3}{|c|}{ VALLE } & \multicolumn{3}{|c|}{ CALI } & \multicolumn{3}{|c|}{ TOTAL } \\
\hline & $\mathrm{N}$ & $\mathrm{n}$ & $\%$ & $\mathrm{~N}$ & $\mathrm{n}$ & $\%$ & $\mathrm{~N}$ & $n$ & $\%$ \\
\hline Tri P1 & 503 & 30 & 6 & 178 & 16 & 9.0 & 681 & 46 & 6.8 \\
\hline Tri 2 & 597 & 32 & 5.4 & 315 & 34 & 10.8 & 912 & 66 & 7.2 \\
\hline Tri 3 & 636 & 15 & 2.4 & 496 & 57 & 11.5 & 1.132 & 72 & 6.4 \\
\hline $\operatorname{Tri} 4$ & 780 & 31 & 4.0 & 459 & 68 & 14.8 & 1.239 & 99 & 8.0 \\
\hline Tri 5 & 832 & 27 & 3.2 & 411 & 70 & 17.0 & 1.243 & 97 & 7.8 \\
\hline Total & 3.612 & 152 & 4.2 & 1.859 & 245 & 13.2 & 5.471 & 397 & 7.2 \\
\hline \multicolumn{3}{|c|}{ Diferencia } & 30 & & & 46.7 & & & \\
\hline \multicolumn{10}{|c|}{$\mathrm{CHI} 2=5,81 \mathrm{P}<0.01$} \\
\hline
\end{tabular}

Tabla 4

CAMBIOS EN LA INCIDENCIA DE LA PREECLAMPSIA

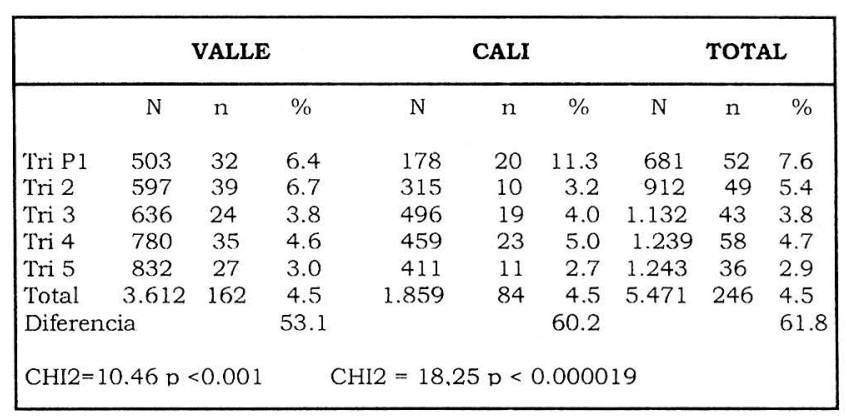

Tabla 5

CAMBIOS EN LA INCIDENCIA DE LA RUPTURA PREMATURA DE MEMBRANAS

\begin{tabular}{|c|c|c|c|c|c|c|c|c|c|}
\hline & \multicolumn{3}{|c|}{ VALLE } & \multicolumn{3}{|c|}{ CALI } & \multicolumn{3}{|c|}{ TOTAL } \\
\hline & $\mathrm{N}$ & $\mathrm{n}$ & $\%$ & $\mathrm{~N}$ & $\mathrm{n}$ & $\%$ & $\mathrm{~N}$ & $\mathrm{n}$ & $\%$ \\
\hline Tri P1 & 503 & 50 & 10.1 & 178 & 10 & 5.6 & 681 & 60 & 8.8 \\
\hline Tri 2 & 597 & 34 & 5.7 & 315 & 9 & 2.9 & 912 & 43 & 4.7 \\
\hline Tri 3 & 636 & 15 & 2.4 & 496 & 16 & 3.2 & 1.132 & 31 & 2.7 \\
\hline $\operatorname{Tri} 4$ & 780 & 27 & 3.5 & 459 & 18 & 3.9 & 1.239 & 45 & 3.6 \\
\hline Tri 5 & 832 & 24 & 2.9 & 411 & 13 & 3.2 & 1.243 & 37 & 3.0 \\
\hline Total & 3.612 & 159 & 4.4 & 1.859 & 67 & 3.6 & 5.471 & 226 & 4.1 \\
\hline \multicolumn{3}{|c|}{ Diferencia } & 71.3 & & & 35.7 & & & 66.0 \\
\hline
\end{tabular}


Tabla 6

CAMBIOS EN LA INCIDENCIA DE LA AMENAZA DE PARTO PREMATURO

\begin{tabular}{|c|c|c|c|c|c|c|c|c|c|}
\hline \multicolumn{4}{|c|}{ VALLE } & \multicolumn{3}{|c|}{ CALI } & \multicolumn{3}{|c|}{ TOTAL } \\
\hline & $\mathrm{N}$ & $\mathrm{n}$ & $\%$ & $\mathrm{~N}$ & $\mathrm{n}$ & $\%$ & $\mathrm{~N}$ & $\mathrm{n}$ & $\%$ \\
\hline Tri Pl & 503 & 29 & 5.8 & 178 & 8 & 4.5 & 681 & 37 & 5.4 \\
\hline Tri 2 & 597 & 24 & 4.0 & 315 & 10 & 3.2 & 912 & 34 & 3.7 \\
\hline Tri 3 & 636 & 37 & 5.8 & 496 & 19 & 3.8 & 1.132 & 56 & 4.9 \\
\hline Tri 4 & 780 & 57 & 7.3 & 459 & 10 & 2.2 & 1.239 & 67 & 5.4 \\
\hline Tri 5 & 832 & 23 & 2.8 & 411 & 10 & 2.4 & 1.243 & 33 & 2.7 \\
\hline Total & 3.612 & 177 & 4.9 & 1.859 & 58 & 3.1 & 5.471 & 235 & 4.3 \\
\hline \multicolumn{3}{|c|}{ Diferencia } & 51.7 & & & 46.7 & & & 50 \\
\hline \multicolumn{10}{|c|}{$\mathrm{CHI} 2=$ Corrección de Yates $6.76 \mathrm{p}<0.0093$} \\
\hline
\end{tabular}

Tabla 7

\section{CAMBIOS EN LA INCIDENCIA DEL PARTO PREMATURO}

\begin{tabular}{|c|c|c|c|c|c|c|c|c|c|}
\hline & \multicolumn{3}{|c|}{ VALLE } & \multicolumn{3}{|c|}{ CALI } & \multicolumn{3}{|c|}{ TOTAL } \\
\hline & $\mathrm{N}$ & $\mathrm{n}$ & $\%$ & $\mathrm{~N}$ & $\mathrm{n}$ & $\%$ & $\mathrm{~N}$ & $\mathrm{n}$ & $\%$ \\
\hline Tri P1 & 503 & 66 & 13.2 & 178 & 15 & 8.5 & 681 & 81 & 11.9 \\
\hline Tri 2 & 597 & 69 & 11.6 & 315 & 25 & 7.9 & 912 & 94 & 10.3 \\
\hline Tri 3 & 636 & 40 & 6.3 & 496 & 26 & 5.2 & 1.132 & 66 & 5.8 \\
\hline Tri 4 & 780 & 50 & 6.4 & 459 & 22 & 4.8 & 1.239 & 72 & 5.8 \\
\hline Tri 5 & 832 & 34 & 4.1 & 411 & 20 & 4.9 & 1.243 & 54 & 4.3 \\
\hline Total & 3.612 & 278 & 7.7 & 1.859 & 108 & 5.8 & 5.471 & 386 & 7.0 \\
\hline \multicolumn{3}{|c|}{ Diferencia } & 41.6 & & & 42.4 & & & 63.9 \\
\hline \multicolumn{4}{|c|}{$\begin{array}{l}\text { CHI2 = Correción de Yates } \\
35.6 p<0.000000\end{array}$} & \multicolumn{3}{|c|}{$\begin{array}{l}\mathrm{CHI} 2=2.22 \\
\text { N. S. p }<0.13\end{array}$} & & & \\
\hline
\end{tabular}

Tabla 8

CAMBIOS EN LA INCIDENCIA DE SINDROMES DE DIFICULTAD RESPIRATORIA

\begin{tabular}{|c|c|c|c|c|c|c|c|c|c|}
\hline & \multicolumn{3}{|c|}{ VALLE } & \multicolumn{3}{|c|}{ CALI } & \multicolumn{3}{|c|}{ TOTAL } \\
\hline & $\mathrm{N}$ & $\mathrm{n}$ & $\%$ & $\mathrm{~N}$ & $\mathrm{n}$ & $\%$ & $\mathrm{~N}$ & $\mathrm{n}$ & $\%$ \\
\hline Tri P1 & 503 & 11 & 2.1 & 178 & 2 & 1.1 & 681 & 13 & 1.9 \\
\hline Tri 2 & 597 & 17 & 2.8 & 315 & 1 & 0.3 & 912 & 18 & 1.9 \\
\hline Tri 3 & 636 & 9 & 1.4 & 496 & 4 & 0.8 & 1.132 & 13 & 1.1 \\
\hline Tri 4 & 780 & 17 & 2.2 & 459 & 5 & 1.1 & 1.239 & 22 & 1.8 \\
\hline Tri 5 & 832 & 6 & 0.7 & 411 & 1 & 0.2 & 1.243 & 7 & 0.6 \\
\hline Total & 3.612 & 61 & 1.7 & 1.859 & 13 & 0.7 & 5.471 & 74 & 1.4 \\
\hline \multicolumn{3}{|c|}{ Diferencia } & 19 & & & 81.8 & & & 68.4 \\
\hline \multicolumn{10}{|c|}{$\mathrm{CH} 2=5,36 \mathrm{P}<0.02$} \\
\hline
\end{tabular}

Tabla 9

CAMBIOS EN LA TASA MORTALIDAD FETAL EN EL VALLE

\begin{tabular}{|lrrr|}
\hline & N & n & $\% 0$ \\
\hline Tri P1 & 503 & 10 & 20.0 \\
Tri 2 & 597 & 5 & 9.0 \\
Tri 3 & 636 & 6 & 9.4 \\
Tri 4 & 780 & 7 & 9.0 \\
Tri 5 & 832 & 1 & 1.0 \\
Total & 3.612 & 29 & 8.0 \\
Diferencia & & & $95 \%$ \\
CHI2 = & & \\
\hline
\end{tabular}

Otros síndromes de dificultad respiratoria en el recién nacido disminuyeron en el Valle 19\%. Tabla 8. Gráfica 8. En Cali y el Valle disminuyeron los niños de bajo peso.

La mortalidad perinatal disminuyó a expensas de la mortalidad fetal que disminuyó $95 \%$ en el Valle. Tabla 9. Gráfica 9.

En Cali disminuyó aunque no de manera tan notable por las serias dificultades que tuvo el programa sobre todo en las instituciones de la comuna 14 por déficit de personal, por falta de pago oportuno e inestabilidad laboral.

\section{Muertes maternas}

Durante el desarrollo del programa no se presentaron muertes maternas atribuibles al control prenatal; hubo una muerte en el Valle atribuible a manejo de nivel II.

En la comuna 14 de Cali en 1996 antes de este programa, ocurrieron cuatro muertes por preeclampsia.

En 1997 no hubo muertes por esta patología.

Según Salazar en revisión de las muertes maternas en Cali durante 10 años 1986 - 1995 más o menos la mitad de las muertes ocurrieron en gestantes con control prenatal. (2).

Es así como en la Ciudad de Cali a partir de 1995, hemos participado junto con otras estrategias de la Secretaría en otras comunas de la Ciudad, en la disminución de un $25 \%$ en la tasa de mortalidad materna.

Debemos resaltar que estas tasas estaban estacionarias durante los 10 años anteriores y que no se trata de subregistro pues es el mismo equipo de búsqueda, vigilancia y análisis que tiene la Secretaría de Salud.

\section{Discusión}

De acuerdo con los resultados presentados que confirman los encontrados en investigación previa, no queda duda de los beneficios de uncontrol prenatal con calidad.

En la medida que se incrementó la detección oportuna de riesgos y algunas patologías como la anemia, infecciones urinarias, infecciones vaginales, etc, se mejoró su tratamiento; esto incidió en la disminución de otras patologías como la amenaza de parto prematuro, el parto prematuro, la pre-eclampsia y la morbilidad y mortalidad del recién nacido con la consiguiente disminución de costos sociales y costos hospitalarios de los niveles II y III de atención.

La inversión en tecnologías básicas y de relativo bajo costo, como consultorios con privacidad dotados con la ropa necesaria y demás elementos para un examen completo incluido el ginecológico, con asignación del tiempo adecuado para la consulta médica (mínimo media hora para la primera consulta) y la actualización permanente del conocimiento médico permiten garantizar los resultados presentados.

Con la metodología de investigación - acción - participación utilizada, el personal asistencial y directivo analizan las deficiencias y toman los correctivos pertinentes.

Se ha logrado superar creencias que han hecho carrera dentro del personal y que creemos inciden directamente en la baja calidad de los servicios. Mencionaremos algunas de ellas: 


\section{a. "El control prenatal es muy sencillo. Sólo basta con medir barrigas"}

Hemos detectado algunos factores que están reforzando esta creencia.

1. Independientemente de las escuelas o facultades de donde egresa el personal, un alto porcentaje no le da importancia al control prenatal. No solo realizan anamnesis incompletas sino que no examinan a la gestante.

2. Al ingresar a trabajar a las instituciones no reciben inducción al cargo, no es frecuente la capacitación en servicio, la actualización ni la unificación de criterios.

3. Las condiciones inadecuadas de los consultorios no permiten la labor del médico. Generalmente los elementos que se necesitan son sencillos y de bajo costo como se ha detectado en otros estudios. (5-8).

4. La política de personal en el país es desestimulante. Los agentes de Salud se encuentran mal remunerados, con pagos atrasados varios meses, sin estabilidad laboral y muchas veces sin seguridad social. Cuando se paga por tarifas estas son irrisorias y lo que se dice es hagan volumen para compensar.

b) "Es que las gestantes no se dejan hacer examen ginecológico"

"Cualquier sangrado u otra complicación culparán al médico."

En la práctica no se realiza especuloscopia a las gestantes. Para realizarlo es necesario conocer los factores culturales y la percepción que las usuarias tienen de los servicios (9-10).

Nosotros lo logramos sin violentarlas, explicándoles, la auxiliar de enfermería en su entrevista previa a la consulta médica, el procedimiento, así como la necesidad de hacerlo para la evolución normal del embarazo.

Teniendo además las condiciones adecuadas en el consultorio para realizarlo de manera respetuosa, con el acompañamiento, si es necesario, de la auxiliar de enfermería.

Comunicando seguridad y teniendo criterios uniformes entre el grupo de personas que la atienden, así como procurando la participación de las gestantes en el seguimiento de su embarazo (vigilancia de incremento de peso, curvas de altura uterina, dudas).

C) "Es que las gestantes no saben el peso previo". No saben la última regla....

A la mayoría de las gestantes no se les lleva su gráfica de ganancia de peso porque suponemos que no saben su peso previo.

Lo real es todo lo contrario; es el menor porcentaje las que no lo saben y para ellas existen otras tablas de peso y talla según edad gestacional. Lo importante es darle el valor que tiene la vigilancia del peso con elementos simples como incremento no mayor de 2 Kilos por mes. Hemos observado casos de muerte materna en control de alto riesgo por especialistas que incrementan 5 y 7 Kilos en un mes y no se detecta el riesgo.

d) "El control prenatal que se realiza en mi institución si es bueno. Lo que pasa es que las gestantes son desnutridas, analfabetas y consultan tardiamente".

Actitud frecuente entre los directivos.
Ya hemos demostrado que si bien estas características son muy importantes, es necesario evaluar las instituciones con indicadores válidos que permitan conocer la magnitud del problema y sus causas así como realizar investigaciones operacionales que permitan identificar las maneras más efectivas de mejorar los servicios. (11-12)

Si las instituciones garantizan la calidad de su control y aplican las normas de subsidio vigentes para las más desprotegidas son muchas las muertes maternas y fetales que se evitan.

Pero no sólo las muertes que se evitan sino que esas madres mejoran su salud y por ende las condiciones del recién nacido.

Si Calidad corresponde a conformidad con las normas (13) estas no son absolutas sino que varían de una sociedad a otra de acuerdo a los tres componentes de la atención en salud como son el ambiente físico dentro del cual se proporciona la atención cuyas características las hacen aceptables o deseables y que varían de una comunidad a otra; el manejo de la relación interpersonal; considerando los valores que incorporan la cultura y las costumbres locales que determinan lo que es correcto, propio o moral en la relación y la atención técnica, que debe tener presente que en muchos lugares puede existir un sistema nativo y el otro "occidental".

$\mathrm{Al}$ producir las normas, es necesario implementarlas, proveer los recursos y condiciones adecuadas para ejecutarlas, seguirlas, controlarlas y evaluarlas, para que sean enriquecidas o modificadas de acuerdo con las características locales (14).

En el sistema actual de atención a la salud de autofinanciación y competencia por el mercado, no sólo por la exigencia de la ley 100 sino por economía es indispensable la garantía de la calidad del servicio (15).

Para esta garantía se requiere diseñar el sistema de monitoreo del cumplimiento de las normas con base en los resultados que se requieren obtener. Existen experiencias con un sistema computarizado de la historia clínica que no logran mejorar la mortalidad materna y perinatal posiblemente porque no incluye todos los parámetros necesarios para el cambio (16).

En este momento el papel de las Sociedades Científicas es de gran valor pues como entidades comprometidas fundamentalmente con el bienestar de la población a través de la investigación y desarrollo científico, pueden de manera imparcial promover la mejoría de la calidad de los servicios y que ésta sea medida con indicadores de bienestar real, de enfermedad y muerte y de esta forma certificar los esfuerzos que las instituciones realizan para garantizar la calidad de los servicios que deben hacerse no solo por cumplir con la ley 100 sino por economía. Estos programas con tecnología de bajo costo no solo son financiables sino rentables al disminuir costos hospitalarios.

También es de fundamental importancia la participación de las usuarias capaces de interpretar y reconocer los indicadores de resultados en la salud (17).

En conclusión al evidenciar la manera inapropiada y de baja calidad como se realizaba el control prenatal en 22 instituciones del departamento del Valle, se estableció un Programa de Mejoramiento Continuo con partici- 
pación del personal administrativo, asistencial y ginecoobstetras con funciones de capacitación, asesoría y control y con el modelo de monitoreo del proceso de atención diseñado, o que produjo una notable mejoría en la salud de madres y recién nacidos confirmando no solo los hallazgos de investigación previa sino que es posible realizarlo en instituciones de diferentes características.

La metodología empleada coincide con las recomendaciones de un estudio colaborativo nacional en China en 1996 (18).

\section{Agradecimientos}

A las instituciones participantes de la Secretaría de Salud Municipal, Secretaría de Salud Departamental, Universidad del Valle, al personal administrativo y asistencial que con su dedicación y valiosa colaboración han contribuido a rescatar la importancia de un buen control prenatal.

\section{BIBLIOGRAFIA}

1. O.P.S. Las Condiciones de Salud en las Américas. Edición 1994. Publicación Científica 549.

2. Salazar Ana. Mortalidad Materna en Cali - tesis magister en Salud Pública.

3. Pazmiño de Osorio Sonia. Mejoramiento Continuo de la Atención Prenatal en instituciones de la Secretaría de Salud y Seguro Social de Cali. Revista Colombiana de Obstetricia y Ginecología. Enero Marzo 1997; 48(1): 39.

4. Gómez Olmedo- M., Delgado Rodríguez M y col. Prenatal care and prevention of preterm birth. A case study in southern spain . Eur J Epidemiol. 1996; 12(1): 37-44.

5. Situation analyses of emergency obstetric care: examples from eleven operations research projects inwest Africa- The prevention of Maternal mortality network. Soc Sci Med 1995; 40(5): 657-667.

6. Gilson L., Magomi M., M-Kangaa E. The structural quality of tanzanian. Primary health facilitie Bull - World - Health - Organ, 1995; 73 (1): 105-114.

7. Barennes H., Tahi FM. No solution for neonatal mortality in sub Saharan Africa? Evaluation and perspectives in the urban environment of Niamey, Niger Sante, 1995; 5(6): 335-340.

8. Iliff PJ., Bonduelle MM., Grupta V. Changing stillbirht rate: Quality of care or quality of patients? Cent Afr J Med 1996; 42(4): 89-92.

9. Bhardwaj N., Hassan SB., Zaheer M. Maternal care receptivity and its relation to perinatal and neonatal mortality. A rural study Indian Pediatr. 1995; 32(4): 416-423.
10. Chiwuzie J., Braimoh S., Unuigbe J., Olumeko P. Causes of Maternal Mortality in a semi - urban Nigerian setting World - Health Forum 1995; 16(4): 405-408.

11. Campbell O., Koblinsky M., Taylor P. Off to a rapid start: appraising maternal mortality and services. Int J Gynaecol Obstet. 1995; 48: 533552.

12. Berg CJ. Prenatal care in developing countries: the World Health. Organization Technical working groupon antenatal care. J Am Med womens Assoc 1995; 50(5): 182-186.

13. Donabedian Avedis. La dimensión internacional de la evaluación y garantía de la calidad. Salud Pública. Mox 1990; 32: 113-117.

14. Buckley ER. The use of standard settin to improve maternity care. Nurs - Times, 1995; 91(31); 32-33.

15. Ley $100-1993$ y decreto 2174 de 1996 por el cual se organiza el sistema obligatorio de garantía de calidad del sistema de seguridad social.

16. Testa; Zanre - YZ., Kone B., Lankovande J., Sondo B. Diffilculties in quality of health valuations in the context of an African hospital: experience of the Ginecology - Obstetrics Department of the nacional hospital center of Ovagadougou, Burquina Faso Med Trop Mars 1996; 56(2): 151-155.

17. Conroy CB. Policy change and its application to safe motherhood programming Int J Gynaecol Obstet. 1995; 48: 5129-5137.

18. Xiang X., Wang L., Xu L. Chung - Hua -Fu Chan- ko - Tsa-Chih 1996; 31(3): 138-141. 